\title{
Relapse of respiratory insufficiency one year after organising pneumonia
}

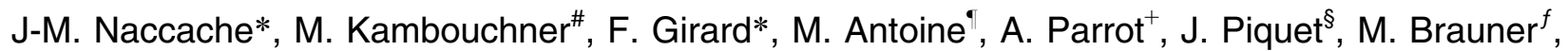 \\ D. Valeyre*
}

\section{Case report}

A 66-yr-old nonsmoker female presented in June 1998 with a 3-week history of progressive dyspnoea. Her past medical history was noteworthy for idiopathic bilateral uveitis without sequelae between 1978 and 1993, hypercholesterolaemia and angina pectoris. Her medication included fluvastatin, which started in June $1997\left(60 \mathrm{mg} \cdot \mathrm{day}^{-1}\right)$, trinitrin patch and acetylsalicylic acid. She had no particular work-related or environmental dust exposure. Examination of the chest revealed bilateral basal crackles. There was no digital clubbing or extrapulmonary signs. At admission, chest radiograph and thoracic computed tomography (CT) scan (fig. 1a) revealed patchy consolidations, predominantly involving the bilateral lower lobes.

Arterial blood gas analysis under supplemental oxygen $\left(6 \mathrm{~L} \cdot \mathrm{min}^{-1}\right)$ revealed a partial pressure of oxygen of $8.3 \mathrm{kPa}$ $(62 \mathrm{mmHg})$, a partial pressure of carbon dioxide of $4.3 \mathrm{kPa}$ $(32 \mathrm{mmHg})$ and a $\mathrm{pH}$ of 7.46 . As a result of rapidly progressive respiratory failure, she was admitted to the intensive care unit. Bronchoalveolar lavage (BAL) analysis showed a $140 \times 10^{6}$ cells $\cdot \mathrm{L}^{-1}$ increased lymphocyte rate $(38 \%)$, low CD4/CD8 ratio $(0.18)$, increased neutrophil rate $(17 \%), 44 \%$ of macrophages and $1 \%$ of eosinophils. Transbronchial lung biopsy, including 20 alveoli, revealed typical lesions of organising pneumonia (fig. 2a). Minor salivary gland biopsy specimens found no sign of Sjögren's syndrome. Antinuclear antibodies were positive at a titre of 1:50 of speckled pattern; anti-JO1 antibodies were positive. Neither clinical nor biological signs of muscular involvement were found. Fluvastatin was stopped.

Oral steroid therapy was initiated $\left(1 \mathrm{mg} \cdot \mathrm{kg}^{-1} \cdot \mathrm{day}^{-1}\right.$ prednisone) and was progressively reduced. The evolution was frankly favourable assessed by clinical symptoms, radiology (fig. 1b) and pulmonary function tests (PFT).

In September 1999, whilst the corticosteroid dose was tapered to $7 \mathrm{mg} \cdot \mathrm{day}^{-1}$, the patient experienced relapsing severe respiratory insufficiency and basal opacities on the chest radiograph. Despite 2 months of high-dose prednisone $\left(1 \mathrm{mg} \cdot \mathrm{kg}^{-1} \cdot \mathrm{day}^{-1}\right)$, no improvement was obtained. Physical examination revealed inspiratory crackles without digital clubbing or extrapulmonary signs. A chest radiograph showed bilateral pulmonary retraction and reticulonodular opacities that were predominant in the lower lobes. PFT revealed a restrictive ventilatory defect, decreased carbon monoxide diffusing capacity of the lung and arterial hypoxaemia necessitating continuous oxygenotherapy. AntiJO1 antibodies were positive without muscular involvement. BAL analysis showed $170 \times 10^{6}$ cells $\cdot \mathrm{L}^{-1}$ with $84 \%$ of macrophages, $14 \%$ of lymphocytes (CD4/CD8: 0.22 ), $1.5 \%$ of neutrophils and $0.5 \%$ of eosinophils. Thoracic high-resolution CT scans were performed (fig. 1c) and the finding resulted in the patient undergoing an open lung biopsy (OLB).

Depts of *Pulmonary Medicine, ${ }^{\#}$ Pathology, and ${ }^{f}$ Radiology, Avicenne University Hospital, Assistance Publique/Hôpitaux de Paris, Bobigny, and Depts of ${ }^{\top}$ Pathology and ${ }^{+}$Pulmonary Medicine, Tenon University Hospital, Assistance Publique/Hôpitaux de Paris, Paris, and ${ }^{\S}$ Dept of Pulmonary Medicine, Montfermeil Intercommunal Hospital, Montfermeil, France. 

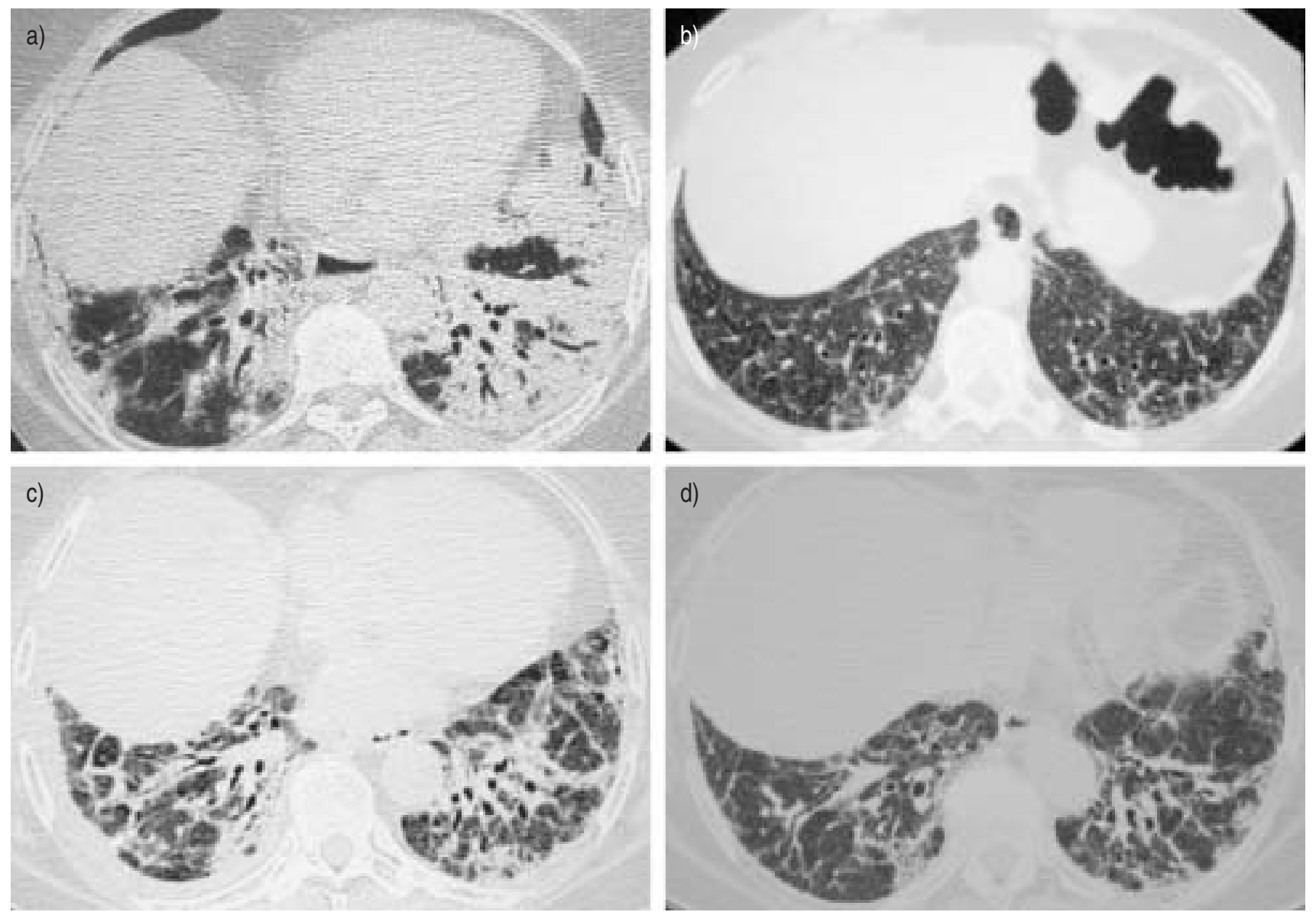

Fig. 1.-Computed tomography (CT) scans of 1-mm thickness at lung window. a) CT scan revealed irregularly marginated patchy alveolar consolidations with an air bronchogram. The lesions were bilateral and predominantly involved the lower lobes. b) After 1 yr, the consolidations disappeared almost completely. CT scan revealed some linear opacities of irregular thickness. c) CT scan from September 1999. d) CT scan revealed the same lesions with some improvement in May 2003.
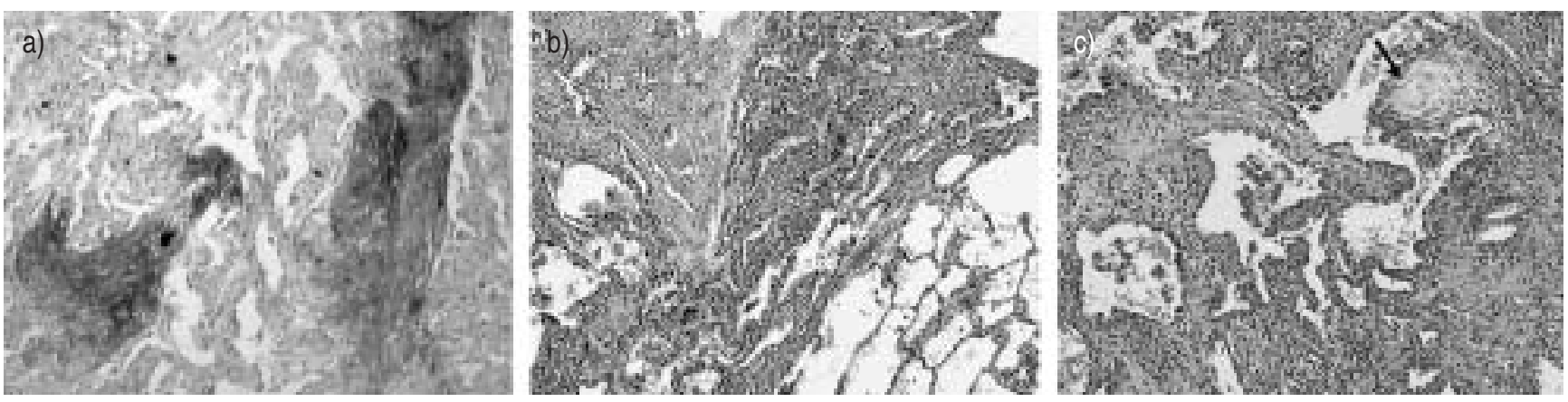

Fig. 2.-a) Transbronchial biopsy showing elongated serpiginous plugs of proliferating fibroblasts filling the airspaces, extending along and branching within alveolar ducts. b, c) Results of open lung biopsy.

\section{BEFORE TURNING THE PAGE, INTERPRET THE CT SCANS AND THE LUNG BIOPSY PATHOLOGY.}




\section{Interpretation}

\section{Thoracic high-resolution computed tomography}

Figure 1c revealed peribronchovascular thickening and septal reticulations in the lower lobes, with distorted linear opacities and some bronchial distortion.

\section{Pathology}

Two specimens were obtained by OLB $(3.5 \times 2 \mathrm{~cm}$ and $4.5 \times 2 \mathrm{~cm}$ ) performed on the right side, in the lower and upper lobe, respectively. Histological sections showed interstitial fibrosis, consistent with usual interstitial pneumonia (UIP), characterised by a nonuniform appearance, with alternating zones of normal lung directly close to zones of active fibrosis of alveolar walls comprising inflammatory cells (fig. 2b). A higher magnification view (fig. 2c) showed areas of honeycomb changes, mild inflammatory infiltrate and few fibroblastic foci (arrow).

Diagnosis: "Usual interstitial pneumonia secondary to fluvastatininduced organising pneumonia".

\section{Clinical course}

In January 2000, treatment combining aziathioprine $\left(2 \mathrm{mg} \cdot \mathrm{kg}^{-1} \cdot \mathrm{day}^{-1}\right)$ and prednisone $\left(7 \mathrm{mg} \cdot \mathrm{day}^{-1}\right)$ was initiated. During the following weeks, a dramatic improvement was observed, and this improvement was maintained until June 2003, despite the withdrawal of steroids and azathioprine for 3 months. There was no sign of connective tissue disorder. Chest radiograph and $\mathrm{CT}$ showed a marked improvement of parenchymal lesions, including bronchiectasis (fig. 1d). PFT were also improved and oxygen therapy was discontinued (fig. 3).

\section{Discussion}

This case is a new report of statin-induced organising pneumonia, in which fluvastatin was implicated for the first time. The severity of respiratory failure, the evidence of a further evolution to UIP and the presence of anti-JO1 antibodies were remarkable. Moreover, the removal of fluvastatine and the introduction of azathiopine lead to dramatic improvement and long-term stabilisation.

Several reasons suggest that organising pneumonia was induced by fluvastatin in this study. First, treatment with fluvastatin and the occurrence of organising pneumonia were undoubtedly time related. Secondly, there was no evidence of any other known cause of organising pneumonia [1]. Particularly, the absence of any systemic manifestation during 5 yrs makes the hypothesis of an associated myositis highly improbable, despite the presence of anti-JO1. Finally, various interstitial lung diseases, including organising pneumonia, have been described with 3-hydroxyl-3-methylglutaryl (HMG)-CoA reductase inhibitors [2-7].

Several cases of interstitial pneumonitis induced by statins have been described. The statins responsible were lovastatin $[2,4]$, simvastatin [3-7] and pravastatin [4, 5]. Only a few cases had histological documentation. One case with organising pneumonia had a life-threatening syndrome with favourable outcome under prednisone therapy [2]. The other cases had hypersensitivity pneumonitis pattern [5], nonspecific interstitial

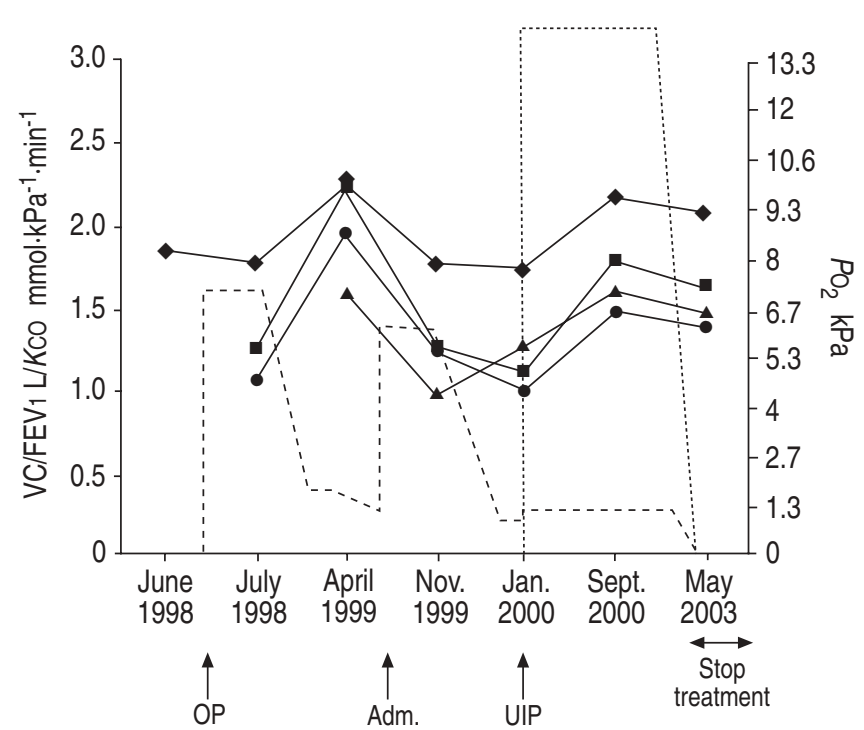

Fig. 3.-Evolution of pulmonary function in the studied patient. Supplemental oxygen at $6 \mathrm{~L} \cdot \mathrm{min}^{-1}$ was applied for the first blood gas analysis. The subsequent blood gas analyses were made without supplemental oxygen. VC: vital capacity (ם); FEV1: forced expiratory volume in one second ( $)$; KCO: carbon monoxide transfer coefficient $(\boldsymbol{\Delta}) ; \mathrm{PO}_{2}$ : partial pressure of oxygen $(\bullet)$; OP: organising pneumonia, diagnosed in June 1998; Adm.: admission to the current authors' unit (Dept of Respiratory Medicine, Avicenne University Hospital, Bobigny, France); UIP: usual interstitial pneumonia, diagnosed in January 2000; Stop treatment: treatment was stopped for 3 months. - - - -: prednisone dose ranging from $60 \mathrm{mg} \cdot \mathrm{day}^{-1}$ after OP diagnosis, which was progressively reduced to 12 and then $7 \mathrm{mg} \cdot \mathrm{day}^{-1}$, and increased to $50 \mathrm{mg} \cdot \mathrm{day}^{-1}$ and subsequently tapered to $5 \mathrm{mg} \cdot \mathrm{day}^{-1}$, until combined treatment with aziathioprine when the dose was $7 \mathrm{mg} \cdot$ day $^{-1} ; \ldots \ldots$ : azathioprine at a dose of $125 \mathrm{mg} \cdot \mathrm{day}^{-1}$.

pneumonia pattern [7] or pulmonary fibrosis without more precision $[3,6]$. The outcome was favourable for all but one case, following statin discontinuation with or without corticosteroid and/or immunosuppressive treatment. The fatal case had pulmonary fibrosis without specification about histological pattern on post mortem examination [3].

The most singular aspect in the current authors' case was the severity of initial presentation of organising pneumonia and the secondary occurrence of UIP 1 yr later. Organising pneumonia is usually considered to have a benign course. Life-threatening variants of this syndrome have been described $[2,8]$, with possible evolution to pulmonary fibrosis or death. Interpretation of such reports is unclear. Whether organising pneumonia really progresses to UIP, or is an associated lesion with another chronic disease (i.e. UIP), is under discussion [9]. CoHEN et al. [8] reported 10 cases of rapidly progressive organising pneumonia. Two cases had organising pneumonia without other lesions on a first OLB, and had UIP in a secondary OLB or post mortem examination. Interestingly, and consistent with the current authors' study, one of these two patients developed UIP 7 months after the initial remission of organising pneumonia under corticosteroid, but, finally, occurrence of UIP rapidly led to death. In the current case, the respective histopathological diagnosis of organising pneumonia and UIP were very typical. Severe pulmonary failure improved after the removal of statin therapy associated with immunosuppressive treatment, and this improvement was stable, despite discontinuing treatment.

The favourable outcome of UIP under aziathioprine and corticosteroid represents another particularity. UIP is the histopathological substratum of idiopathic pulmonary fibrosis 
(IPF) [10]. Prognosis of IPF is poor, with a median survival of 24-36 months. Thus, the rapidly favourable outcome of the current patient after this regimen was particularly remarkable in this context. The stabilisation of the respiratory condition 3 yrs later, despite the cessation of immunosuppressive treatment, was probably linked to a different pathogenesis than in IPF, since only very few patients with IPF respond favourably to immunosuppressive treatments.

The last particular aspect was the positivity of anti-JO1 antibodies without muscular involvement during a 5-yr follow-up. A statin-induced dysimmune process has been suspected in some patients. Anti-nuclear antibodies and/or systemic reactions sometimes associated with pulmonary involvement have been reported under statin treatment [3-5]. Extractable nuclear antigen testing was negative. Anti-JO1 antibodies are auto-antibodies anti-histidyl-tRNA synthetase. They are the most frequent myositis-specific autoantibodies. They are found in $20-30 \%$ of patients with myositis, and in $50-100 \%$ of patients with interstitial lung disease (ILD)-associated myositis [11].

ILD that is associated with positive anti-JO1 antibodies without muscle involvement is rare. SAUTY et al. [12] reported four cases with this clinical pattern. One of these cases developed myositis 9 months after the onset of ILD. Interestingly, one patient had ischaemic cardiopathy, but her treatment was not mentioned. The differential cell count of BAL of this patient showed $11 \%$ of eosinophils, which could suggest an allergic process. In the four cases, histological findings of transbronchial lung biopsy disclosed a pattern of nonspecific interstitial pneumonitis with a CD8+ lymphocytosis on BAL. In three cases, immunosuppressive therapy was commenced with a favourable outcome. Conversely, myalgia, myopathy and dermatomyositis are classical side-effects of HMG-CoA reductase inhibitors [3, 5, 13]. Few cases of patients with muscular involvement are associated with ILD, immune reactions and positive antinuclear antibodies, but without anti-JO1 [3, 5].

In conclusion, the current authors' case emphasises the possible occurrence of a new and severe variant of statininduced interstitial lung disease associated with an immune reaction.

\section{References}

1. Cordier JF. Organising pneumonia. Thorax 2000; 55: 318-328.

2. Nizami IY, Kissner DG, Visscher DW, Dubaybo BA. Idiopathic bronchiolitis obliterans with organizing pneumonia. An acute and life-threatening syndrome. Chest 1995; 108: 271-277.

3. Hill C, Zeitz C, Kirkham B. Dermatomyositis with lung involvement in a patient treated with simvastatin. Aust $N Z$ J Med 1995; 25: 745-746.

4. Liebhaber MI, Wright RS, Gelberg HJ, Dyer Z, Kupperman JL. Polymyalgia, hypersensitivity pneumonitis and other reactions in patients receiving HMG-CoA reductase inhibitors: a report of ten cases. Chest 1999; 115: 886-889.

5. Rudski L, Rabinovitch MA, Danoff D. Systemic immune reactions to HMG-CoA reductase inhibitors. Report of 4 cases and review of the literature. Medicine (Baltimore) 1998; 77: 378-383.

6. De Groot RE, Willems LN, Dijkman JH. Interstitial lung disease with pleural effusion caused by simvastin. J Intern Med 1996; 239: 361-363.

7. Lantuejoul S, Brambilla E, Brambilla C, Devouassoux G. Statin-induced fibrotic nonspecific interstitial pneumonia. Eur Respir J 2002; 19: 577-580.

8. Cohen AJ, King TE Jr, Downey GP. Rapidly progressive bronchiolitis obliterans with organizing pneumonia. Am J Respir Crit Care Med 1994; 149: 1670-1675.

9. Costabel U, Guzman J, Teschler H. Bronchiolitis obliterans with organising pneumonia: outcome. Thorax 1995; 50: S59S64.

10. du Bois RM, Wells AU. Cryptogenic fibrosing alveolitis/ idiopathic pulmonary fibrosis. Eur Respir J Suppl 2001; 32: $43 \mathrm{~s}-55 \mathrm{~s}$.

11. Marie I, Dominique S, Remy-Jardin M, Hatron PY, Hachulla E. Interstitial lung diseases in polymyositis and dermatomyositis. Rev Med Interne 2001; 22: 1083-1096.

12. Sauty A, Rochat T, Schoch OD, et al. Pulmonary fibrosis with predominant CD8 lymphocytic alveolitis and anti-Jo-1 antibodies. Eur Respir J 1997; 10: 2907-2912.

13. Ballantyne CM, Corsini A, Davidson $\mathrm{MH}$, et al. Risk for myopathy with statin therapy in high-risk patients. Arch Intern Med 2003; 163: 553-564. 